\title{
Facile Synthesis of Organometal Perovskite Microcubes of Optical Quality using Polar Antisolvents
}

\author{
Yuelong $\mathrm{Li}^{1,2} \dagger$, Juan F. Galisteo-López $†$, and Hernán Míguez ${ }^{1 *}$ \\ ${ }^{1}$ Instituto de Ciencia de Materiales de Sevilla, Consejo Superior de Investigaciones Científicas (CSIC)-Universidad \\ de Sevilla, C/Américo Vespucio 49, 41092 Sevilla, Spain \\ ${ }^{2}$ Institute of Photoelectronic Thin Film Devices and Technology, Nankai University, \#94 Weijin Road, 30oo71 Tian- \\ jin, P. R. China
}

Supporting Information Placeholder

\begin{abstract}
Herein, we demonstrate a facile synthetic approach producing highly crystalline methylammonium lead bromide perovskite ( $\mathrm{MAPbBr} 3)$ microcubes at room temperature by injecting perovskite precursor solution into a userfriendly antisolvent like isopropanol. Confirmed by X-Ray and electron diffraction, as well as electron microscopy, these $\mathrm{MAPbBr} 3$ microcubes are single crystals, and have perfect cubic structure with size varying between 1-15 $\mu \mathrm{m}$ depending on the added amount of precursor solution. At the same time, the stoichiometry of $\mathrm{MAPbBr} 3$ crystal is proved by EDX analysis. Finally optical analysis carried out by means of laser scanning confocal microscopy evidences how the crystalline quality of the microcubes translates into a homogeneous photoluminescence throughout the cube volume.
\end{abstract}

Most successful solar cell materials crystallize in a cubic structure. Methylammonium lead halide perovskite, already demonstrating itself as an emerging semiconductor with superior properties for photovoltaics, is not an exception. According to the Bravais-Friedel-Harker law, methylammonium lead bromide ( $\mathrm{MAPbBr}$ ) perovskites usually exhibit a cubic unit cell structure with highly symmetrical bonding interactions in three dimensions at room temperature, and thus readily form crystals of cubic shape. [1,2] Up to date, many researches have been conducted to synthesize perovskite nano/microcrystals materials in colloidal chemistry, and in most synthetic routes, the use of long chain alkyl (C8C18) molecules with coordinating functional groups (such as - $\mathrm{COOH},-\mathrm{NH} 2$, etc.) as surfactant ligands is crucial for stabilization, for prevention of aggregation, and for size and shape control of nano/microcrystals. Depending on the length and functional group of surfactants added in the perovskite precursor solution or antisolvent for precipitates, this ligand-assisted reprecipitation process normally leads to specific nano/microcrystals such as wires, rod, platelets, triangles, etc. $[1,3,4,5,6,7,8,9]$ However, it is quite surprising that the synthesis of methylammonium lead halide cubic structures, especially the bromide based one, have been rarely demonstrated. [10]
In this work, a facile synthetic approach is developed to produce highly crystalline methylammonium lead bromide perovskite (MAPbBr3) microcubes at room temperature by injecting perovskite precursor solution into a polar protic antisolvent without any assistance from surfactants or special post-treatments. These MAPbBr3 crystals have perfect cubic shape and constitute single crystals as evidenced by combined scanning (SEM) and transmission (TEM) electron microscopy as well as X-ray diffraction (XRD). Further, the cube size can be varied between 1 and $15 \mu \mathrm{m}$ by controlling the amount of precursor solution added to the polar protic antisolvent in the reaction. Compared with the previously reported procedures, our synthesis does not require the use of toxic non-polar solvents such as chlorobenzene, chloroform or toluene, which are normally used as antisolvents for inducing precipitation of perovskite materials, or the preparation of halide organic surfactants with long ligands. The presence of these macromolecules, or the residual of organic solvents for dissolving them such as octadecene, may create highly insulating barriers which block electronic communication between nano/microcrystals, limiting the usefulness of their assemblies for optoelectronic applications. The optical properties of the fabricated structures are studied by means of laser scanning confocal microscopy (LSCM). This technique allows analyzing the homogeneity of the photoemission in the bulk of the microstructure by collecting the photoluminescence (PL) from small volumes (voxel of $300 \mathrm{x}$ $300 \times 400 \mathrm{~nm}^{3}$ ). This characterization allows for a precise knowledge of their emissive properties which are relevant for evaluating both the quality of the lattice, as PL is highly sensitive to its distortions and defects and their potential applications, as lead halide perovskites are expected to play a key role in optoelectronic devices involving light emission. [11] In particular, single crystals growing into polygonal shapes constitute optical micro-cavities whose potential as micro-lasers has been broadly demonstrated over the past few years. [1,12,13,14,15,16,17,18,19]

In the synthetic procedure herein proposed the $\mathrm{MAPbBr}_{3}$ precursor solution was prepared by dissolving methyl ammonium cations and $\mathrm{PbBr}_{2}$ in a polar DMF solvent where methyl ammonium cations are embedded in the voids of a 
set of corner-sharing $\mathrm{PbX}_{6}$ octahedra to form organicinorganic hybrid $\mathrm{MAPbBr}_{3}$ perovskite. In order to accelerate the nucleation and precipitation process antisolvents has been commonly employed. They play a critical role in extracting the DMF solvent from the perovskite precursor solution as a result of the antisolvent/DMF miscibility. Toxic non-polar solvents such as chlorobenzene, toluene, chloroform and diethyl ether are among those more frequently used. However, according to the similarity principle, DMF, a polar aprotic solvent, should have better miscibility with polar solvents such as simple alcohols, organic nitriles or simple ketones, which could potentially facilitate the preparation of well-crystallized perovskite micro/nanostructured materials. In our work, isopropanol alcohol (IPA), a polar solvent, has been chosen as the standard antisolvent to produce $\mathrm{MAPbBr}_{3}$ microcubes. Typically, $50 \mu \mathrm{L}$ of $40 \mathrm{ot} \% \mathrm{MAP}-$ $\mathrm{bBr}_{3}$ in DMF is rapidly injected into $950 \mu \mathrm{L}$ of IPA solvent with stirring at room temperature, which immediately produces orange precipitates at the bottom. After 1 min stirring, a drop of orange precipitates was coated onto a slide glass and dried at $100{ }^{\circ} \mathrm{C}$ for $10-20$ min. Under the SEM (Fig. 1a) it can be clearly seen that as-prepared perovskite microcubes exhibit a smooth surface and sharp edges with perfect threedimensional (3D) cubic structure as depicted in the inset image, and size from 1-5 $\mu \mathrm{m}$. According to our preliminary tests with different solvents, either polar or non-polar ones, (Figure $\mathrm{S}_{1}$ of Supporting Information), IPA is a preferred choice to attain perovskite micro-structures with perfect cubic shape. Due to the limited miscibility of polar DMF in non-polar antisolvents, residual DMF solvent results in truncated perovskite microcubes, as a consequence of either an etching effect of the remaining DMF in the case of chlorobenzene and toluene, or the presence of unreacted perovskite precursor remains on the surface of perovskites cubes in the case of diethyl ether and chloroform. On the other hand, not every polar solvent, such as acetone or methanol, produces perfect perovskite cubes. Although we observed that acetonitrile gives rise to perfect cubic perovskite, there was always some taint present on the surface. Only IPA among our tested solvents yields $\mathrm{MAPbBr}_{3}$ perovskite microcubes with a smooth surface and sharp edges, which suggests that its miscibility with DMF is appropriate to prevent the contamination of the final microcubes with any kind of impurity.

The concentration effect of the perovskite precursor solution (Figure $\mathrm{S}_{2}$ of Supporting Information) was also investigated, $\mathrm{MAPbBr}_{3}$ microcubes being only achieved with $40 \mathrm{wt} \%$ precursor solution. The rest of concentrations led either to truncated cubes (10wt $\%$ or $20 w t \%)$ or none at all $(1 w t \%)$, evidencing the importance of a concentrated perovskite precursor solution in attaining perfect microcubes. The latter observation indicates that, in order to attain high quality crystallites, the uptake of perovskite precursor during the rapid formation of perovskite microcubes has to be quickly compensated, which would explain why the best results are obtained with highly concentrated perovskite precursor solutions. When the amount of perovskite precursor solution of choice (concentration of $40 w \mathrm{t} \%$ ) added into the antisolvent is varied from $10 \mu \mathrm{L}$ up to $500 \mu \mathrm{L}$, we observe that the average size of $\mathrm{MAPbBr}_{3}$ microcubes can eventually reach about $15 \mu \mathrm{m}$ as long as there is a sufficient amount of precursor solution $(200 \mu \mathrm{L}$ or $500 \mu \mathrm{L})$, although at the expense of widening the size distribution (SEM images of Figure S3a, $\mathrm{S} 3 \mathrm{c}$ of Supporting Information). It can be seen from the X-ray diffraction (XRD) pattern (Fig. 1b) that these $\mathrm{MAPbBr}_{3} \mathrm{mi}$ crocubes have completed conversion from $\mathrm{PbBr}_{2}$ and $\mathrm{MABr}$ to $\mathrm{MAPbBr}_{3}$, with well crystallized structures as indicated from its intense peak of (oo1), (110) and (0o2), without residual peaks from the precursor materials of $\mathrm{PbBr}_{2}$ and $\mathrm{MABr}$. Further, the calculated $d$-spacing of $2.97 \AA$ based on the intense peak of (002) at $2 \theta=30.12 \mathrm{O}$ suggests a cubic lattice structure with a unit cell of $a=5.93 \AA$, which can be indexed assuming cubic symmetry with space group $(\mathrm{Pm} \overline{3} \mathrm{~m})$. As the amount of precursor solution added into the antisolvent increases, the shape of the microcubes is deformed with a slight elongation along the (oo1) direction, resulting in an increased intensity of the (oo1) and (oo2) peaks (Figure S3b of Supporting Information). The selected area electron diffraction (SAED) pattern also shows a high crystalline quality with the same (oo1) facets exposed (Fig. 1c) with measured $d$ spacing of $2.93 \AA$ to plane (200), which is perfectly matched with the XRD results. For the chemical composition of the microcubes, the energy-dispersive X-ray spectroscopy (EDX) analysis presents a $\mathrm{Pb}: \mathrm{Br}$ atomic ratio 1:2.83 (Fig. 1d), which is in good agreement with the 1:3 stoichiometry of a MAP$\mathrm{bBr}_{3}$ crystal.
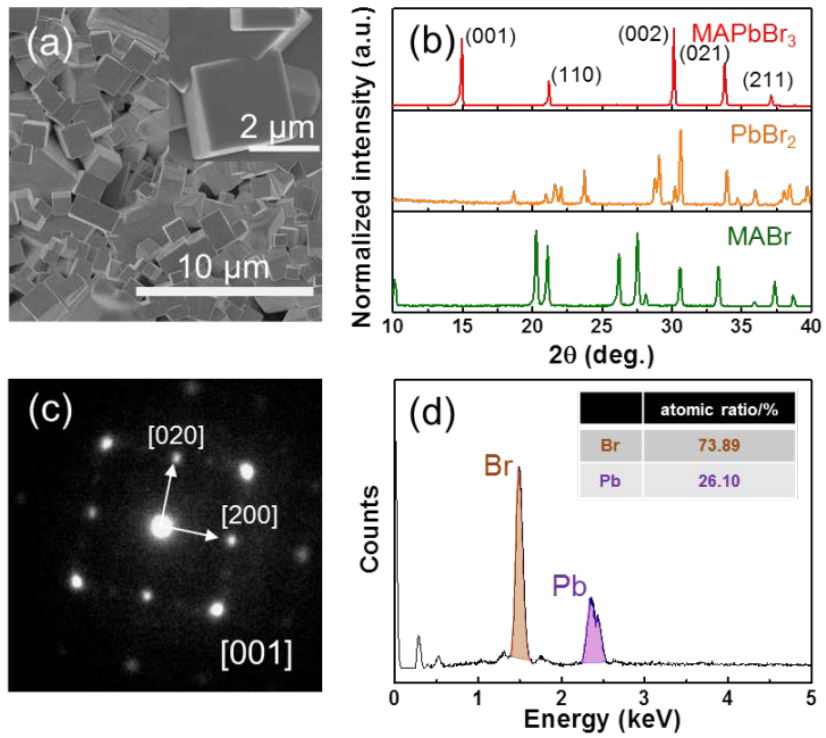

Figure 1. As-prepared $\mathrm{MAPbBr} 3$ microcubes from $50 \mu \mathrm{L}$ precursor solution, characterized by scanning electron microscopy (a) and powder X-ray diffraction (XRD) (b). Selected area electron diffraction pattern of single microcube (c) and its Energy-dispersive X-ray spectroscopy (EDX) spectrum (d). In (b), the XRD patterns of the raw compounds used to prepare the precursor, $\mathrm{PbBr} 2$ and $\mathrm{MABr}$, are also included. The inset in (d) shows the estimated atomic ratio of $\mathrm{Br}$ and $\mathrm{Pb}$ in a microcube.

Studying the emission properties of individual microstructures is essential to evaluate its potential as active components for optoelectronic devices. In order to do so we have studied the described micro-cubes using a LSCM, which allows retrieving information on the homogeneity of the emission at the microscopic level within the volume of the micro-structures containing both intensity as well as spectral information. [20] Figure 2 shows PL images corresponding to different sections of an individual $\mathrm{MAPbI}_{3}$ micro-cube which allow accessing its emissive properties, together with PL 
profiles extracted from them. In these images the outer part of the cube emits more efficiently than the center with the edges showing the highest intensity. This effect is believed to be due to the interaction of the micro-structure with the atmosphere [20] which is known to strongly affect its emissive properties. [21,22,23]



Figure 2. PL intensity profiles (left) and PL images (right panel) collected across different sections of a micro-cube having $2 \mu \mathrm{m}$ sides at three different vertical positions: $\mathrm{z}=0.4$ $\mu \mathrm{m}(\mathrm{a}), \mathrm{z}=1 \mu \mathrm{m}$ (b) and $\mathrm{z}=1.8 \mu \mathrm{m}$ (c). Arrow indicates direction along which profiles are extracted. Scale bars correspond to $1 \mu \mathrm{m}(\mathrm{d})$.

Besides the asymmetry in the PL intensity between edges and center (up to a factor of 1.5), the bulk of the cube presents a highly homogeneous PL intensity as can be seen in the emission profile extracted from a PL image in Fig. $2 b$. This shows how the structural homogeneity of the samples, evidenced by the SEM and TEM analysis, is translated into a high optical quality indicating the absence of defects or imperfections (at least within the spatial resolution limited by optical diffraction in our experiments). Such homogeneity is in contrast with the highly irregular PL distribution previously observed in polycrystalline films of MAPbI3. [24,25]

Finally the spectral properties of micro-cubes of different size were also probed by LSCM. Figure za-b show the evolution of the spectral position of the PL peak as well as its full width at half maximum (FWHM) for micro-cubes having different lateral size. Both magnitudes present a spread of values of ca. $\pm 1.5 \mathrm{~nm}$ but only the former has a clear trend, showing a small blueshift as the cube size increases from below 1 to $10 \mu \mathrm{m}$. These variations are responsible for the spectral differences when we probe a single micro-cube with the confocal microscope and an array of several cubes (from a sample fabricated by injecting $200 \mu \mathrm{l}$ of the MAPbBr3 precursor into the isopropanol solvent) in a conventional PL experiment (Fig. 3c). Spectral variations in the PL of hybrid organic inorganic perovskite crystals depending on their size have been reported [26] for the case of $\mathrm{MAPbI}_{3}$. Such changes comprised the opposite trend to the one observed here, the PL spectra undergoing a blueshift with decreasing crystal size, and were attributed to distortions in the $\mathrm{Pb}-\mathrm{I}-\mathrm{Pb}$ bond as a consequence of crystallite size. [27] A spectral shift in the PL of perovskite crystals due to quantum confinement effects, which would also take place in the opposite direction as the one observed here, can be discarded as the size of the structures studied are well above the Bohr exciton radius reported for this material. [28, 29,30] While a precise knowledge of the origin of the observed spectral shift is lacking at the moment and will demand future work, a plausible explanation could be related to the first mechanism discussed. This would imply the absence of distortions in the smaller micro-cubes, contrary to what has been observed in irregularly shaped crystallites. [26]
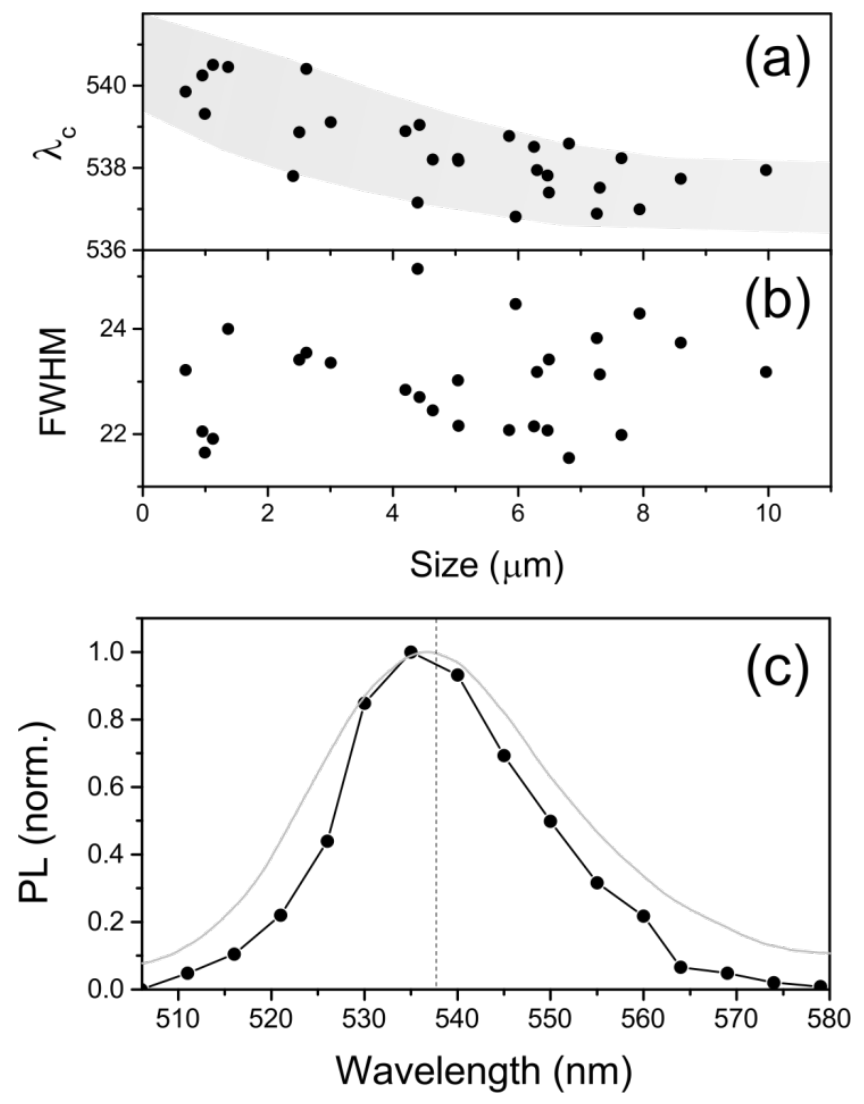

Figure 3. Evolution of PL peak position (a) and FWHM (b) for micro-cubes having different lateral sizes. Grey band is a guide to the eye. (c) PL spectra collected from a single cube using the confocal microscope (black) and from a distribution of cubes using a conventional PL set-up (grey data).

In conclusion, MAPbBr3 microcubes were synthesized by a facile approach where a small amount of $\mathrm{MAPbBr}_{3}$ precursor solution is injected into a user-friendly isopropanol polar antisolvent at room temperature, without assistance of large organic surfactants. XRD and SAED analysis confirm that $\mathrm{MAPbBr}_{3}$ microcubes are single crystals and have a cubic lattice structure with a unit cell of $5.93 \AA$. EDX analysis proves the stoichiometry of $\mathrm{CH}_{3} \mathrm{NH}_{3} \mathrm{PbBr}_{3}$ crystal as well. Optical characterization performed by means of LSCM shows how the previous structural properties translate into a homogenous PL within the crystal bulk, enhanced at its boundaries. The constant emission throughout the cube volume, highly desirable for lighting applications involving this kind of micro-structures, evidences the absence of crystalline defects within the spatial resolution provided by our set-up. Our research could ease the integration of microstructures in 
optoelectronic devices, as the crystallites herein reported present high optical quality and can be readily processed without the need of removing unwanted organic residues from the synthesis.

\section{ASSOCIATED CONTENT}

\section{Supporting Information}

Detailed synthetic procedure and characterization of microcubes samples. SEM images of samples fabricated using different antisolvents. SEM images of samples fabricated using different precursor concentration. SEM images, XRD and cube size histograms for samples fabricated with different amounts of precursor solution. TEM image of micro cube damaged under electron beam.

This material is available free of charge via the Internet at http://pubs.acs.org.

\section{AUTHOR INFORMATION}

\section{Corresponding Author}

h.miguez@csic.es

\section{Author Contributions}

${ }^{\dagger}$ These authors contribute equally.

\section{Notes}

The authors declare no competing financial interests.

\section{ACKNOWLEDGMENT}

Financial support of the European Research Council under the European Union's Seventh Framework Programme (FP7/2007-2013)/ERC grant agreement n³07081 (POLIGHT) and the Spanish Ministry of Economy and Competitiveness under grant MAT2014-54852-R is gratefully acknowledged. YL acknowledges the financial support from the People Programme (Marie Curie Actions) of the European Union's Seventh Framework Programme FP7/2007-2013/ under REA grant agreement $n^{\circ}$ 622533. Juan Luis Ribas at the Centro de Investigación Tecnología e Innovación de la Universidad de Sevilla (CITIUS) is acknowledged for assistance with correlative microscopy expriments.

\section{REFERENCES}

1 Zhang, W.; Peng, L.; Liu, J.; Tang, A.; Hu, J-S.; Yao, J.; Zhao, Y. S. Controlling the Cavity Structures of Two-Photon-Pumped Perovskite Microlasers Adv. Mater. 2016, 28, 4040-4046.

2 Winn, D.; Doherty, M. F. Modeling Crystal Shapes of Organic Materials Grown from Solution AIChE J. 20oo, 46,1348-1367.

3 Ling, Y.; Yuan, Z.; Tian, Y.; Wang, X.; Wang, J. C.; Xin, Y.; Hanson, K.; Ma, B.; Gao, H. Bright Light-Emitting Diodes Based on Organometal Halide Perovskite Nanoplatelets Adv. Mater. 2016, 28, 305-311.
4 Jellicoe, T.C.; Richter, J.M.; Glass, H.F.J.; Maxim, T.; Brady, R.; Dutton, S. E.; Rao, A.; Friend, R.H.; Credgington, D.; Greenham, N. C.; Böhm, M.L. Synthesis and Optical Properties of Lead-Free Cesium Tin Halide Perovskite Nanocrystals J. Amer. Chem. Soc. 2016, 138, 2941-2944.

5 Zhu, F.; Men, L.; Guo, Y.; Zhu, Q.; Bhattacharjee, U.; Goodwin, P.M.; Petrich, J. W.; Smith, E. A.; Vela, J. Shape Evolution and Single Particle Luminescence of Organometal Halide Perovskite Nanocrystals ACS Nano 2015, 9, 2948-2959.

6 Sun, S.; Yuan, D.; Xu, Y.; Wang, A.; Deng, Z. Ligand-Mediated Synthesis of Shape-Controlled Cesium Lead Halide Perovskite Nanocrystals via Reprecipitation Process at Room Temperature ACS Nano 2016, 10, 3648-3657.

7 Zhang, F.; Zhong, H.; Chen, C.; Wu, X-G.; Hu, X.; Huang, H.; Han, J.; Zou, B.; Dong, Y. Brightly Luminescent and Color-Tunable Colloidal $\mathrm{CH}_{3} \mathrm{NH}_{3} \mathrm{PbX}_{3}(\mathrm{X}=\mathrm{Br}, \mathrm{I}, \mathrm{Cl})$ Quantum Dots: Potential Alternatives for Display Technology ACS Nano 2015, 9, 4533-4542.

8 Schmidt, L.C.; Pertegás, A.; González-Carrero, S.; Malinkiewicz, O.; Agouram, S.; Mínguez-Espallargas, G.; Bolink, H.; Galian, R.E.; Pérez-Prieto, J. Nontemplate Synthesis of $\mathrm{CH}_{3} \mathrm{NH}_{3} \mathrm{PbBr} 3$ Perovskite Nanoparticles J. Amer. Chem. Soc. 2014, 136, 850-853.

9 Fu, Y.; Meng, F.; Rowley, M. B.; Thompson, B. J.; Sharer, M. J.; Ma, D.; Hamers, R. J.; Wright, J. C.; Jin, S. Solution Growth of Single Crystal Methylammonium Lead Halide Perovskite Nanostructures for Optoelectronic and Photovoltaic Applications J. Amer. Chem. Soc. 2015, 137, 5810-5818.

$10 \mathrm{Yu}$, J. C.; Kim, D. B.; Jung, E. D.; Lee, B. R.; Song, M.H. HighPerformance Perovskite Light-Emitting Diodes via Morphological Control of Perovskite Films Nanoscale 2016, 8, 7036-7042.

11 Sutherland, B. R. and Sargent, E. H. Perovskite Photonic Sources Nat. Photon. 2016, 10, 295-302.

12 Zhu, H.; Fu, Y.; Meng, F.; Wu, X.; Gong, Z.; Ding, Q.; Gustafsson, M. V.; Trinh, M. T.; Jin, S.; Zhu, X-Y. Lead Halide Perovskite 
Nanowire Lasers with Low Lasing Thresholds and High Quality Factors Nat. Mater. 2015, 14, 636-643.

13 Liu, S.; Sun, W.; Gu, Z.; Wang, K.; Zhang, N.; Xiao, S.; Song, Q. Tailoring the Lasing Modes in $\mathrm{CH}_{3} \mathrm{NH}_{3} \mathrm{PbBr}_{3}$ Perovskite Microplates via Micro-Manipulation RSC Adv. 2016, 6, 50553-50558.

14 Eaton, S. W.; Lai, M.; Gibson, N. A.; Wong, A. B.; Dou, L.; Ma, J.; Wang, L-W.; Leone, S. R.; Yang, P. Lasing in Robust Cesium Lead Halide Perovskite Nanowires Proc. Natl. Acad. Sci. U. S. A. 2016, 113, 1993-1998.

15 Wang, K.; Sun, W.; Li, J.; Gu, Z.; Xiao, S.; Song, Q. Unidirectional Lasing Emissions from $\mathrm{CH}_{3} \mathrm{NH}_{3} \mathrm{PbBr}_{3}$ Perovskite Microdisks ACS Photonics 2016, 6, 1125-1130.

16 Zhang, Q.; Ha, S. T.; Liu, X.; Sum, T. C.; Xiong, Q. RoomTemperature Near-Infrared High-Q Perovskite Whispering-Gallery Planar Nanolasers Nano Lett. 2014, 10, 5995-6001.

17 Liao, Q.; Hu, K.; Zhang, H.; Wang, X.; Yao, J.; Fu, H. Perovskite Microdisk Microlasers Self-Assembled from Solution Adv. Mater. 2015, 27, 3405-3410.

18 Fu, Y.; Zhu, H.; Schrader, A. W.; Liang, D.; Ding, Q.; Joshi, P.; Hwang, L.; Zhu, X-Y.; Jin, S. Nanowire Lasers of Formamidinium Lead Halide Perovskites and Their Stabilized Alloys with Improved Stability Nano Lett. 2016, 16, 1000-1008.

19 Xing, J.; Liu, X. F.; Zhang, Q.; Ha, S. T.; Yuan, Y. W.; Shen, C.; Sum, T. C.; Xiong, Q. Vapor Phase Synthesis of Organometal Halide Perovskite Nanowires for Tunable Room-Temperature Nanolasers Nano Lett. 2015, 15, 4571-5477.

20 Galisteo-López, J.F.; Li, Y.; Míguez, H. Three-Dimensional Optical Tomography and Correlated Elemental Analysis of Hybrid Perovskite Microstructures: An Insight into Defect-Related Lattice Distortion and Photoinduced Ion Migration J. Phys. Chem. Lett. 2016, 7, 5227-5234.

21 Galisteo-López, J. F.; Anaya, M.; Calvo, M. E.; Míguez, H. Environmental Effects on the Photophysics of Organic-Inorganic Halide Perovskites J. Phys. Chem. Lett. 2015, 6, 2200-2205.
22 Tian, Y.; Peter, M.; Unger, E.; Abdellah, M.; Zheng, K.; Pullerits, T.; Yartsev, A.; Sundström, V.; Scheblykin, I. G. Mechanistic Insights into Perovskite Photoluminescence Enhancement: Light Curing with Oxygen Can Boost Yield Thousandfold Phys. Chem. Chem. Phys. 2015, 17, 24978-24987.

23 Fang, H.-H.; Adjokatse, S.; Wei, H.; Yang, J.; Blake, G. R.; Huang, J.; Even, J.; Loi, M. A. Ultrahigh Sensitivity of Methylammonium Lead Tribromide Perovskite Single Crystals to Environmental Gases Sci. Adv. 2016, 2, e1600534.

24 deQuilettes, D. W.; Vorpahl, S. M.; Stranks, S. D.; Nagaoka, H.; Eperon, G. E.; Ziffer, M. E.; Snaith, H. J.; Ginger, D. S. Impact of microstructure on local carrier lifetime in perovskite solar cells Science 2015, 348, 683-686.

25 deQuilettes, D. W.; Zhang, W.; Burlakov, V. M.; Graham, D. J.; Leitjens, T.; Osherov, A.; Bulovic, V.; Snaith, H. J.; Ginger, D. S.; Stranks, S. D. Photo-Induced Halide Redistribution in OrganicInorganic Perovskite Films Nat. Commun. 2016, 7, 11683.

26 D’Innocenzo, V.; Kandada, A. R. S.; De bastiani, M.; Gandini, M.; Petrozza, A. Tuning the Light Emission Properties by Band Gap Engineering in Hybrid Lead Halide Perovskite J. Amer. Chem. Soc. 2016, 136, 17730-17733.

27 Grancini, G.; Marras, S.; Prato, M.; Giannini, C.; Quarti, C.; Angelis, F. D.; Bastiani, M. D.; Eperon, G. E.; Snaith, H. J.; Manna, L.; Petrozza, A. The Impact of the Crystallization Processes on the Structural and Optical Properties of Hybrid Perovskite Films for Photovoltaics J. Phys. Chem. Lett. 2014, 5, 3836-3842.

28 D’Innocenzo, V.; Grancini, G.; Alcocer, M. J. P.; Kandada, A. R. S.; Stranks, S. D.; Lee, M. M.; Lanzani, G.; Snaith, H. J.; Petrozza, A. Excitons Versus Free Charges in Organo-Lead Tri-Halide Perovskites Nat. Commun. 2014, 5, 3585.

29 Menéndez-Proupin, E.; Palacios, P.; Wahnón, P.; Conesa, J. C. Self-Consistent Relativistic Band Structure of the $\mathrm{CH}_{3} \mathrm{NH}_{3} \mathrm{PbI}_{3}$ Perovskite Phys. Rev. B 2014, 90, 045207. 
3o Ma, J.; Wang, L.-W. Nanoscale Charge Localization Induced by Random Orientations of Organic Molecules in Hybrid Perovskite $\mathrm{CH}_{3} \mathrm{NH}_{3} \mathrm{PbI}_{3}$ Nano Lett. 2015, 15, 248-253. 